\title{
Depth and Stanley depth of the edge ideals of the powers of paths and cycles
}

\author{
Zahid Iqbal and Muhammad Ishaq
}

\begin{abstract}
Let $k$ be a positive integer. We compute depth and Stanley depth of the quotient ring of the edge ideal associated to the $k^{t h}$ power of a path on $n$ vertices. We show that both depth and Stanley depth have the same values and can be given in terms of $k$ and $n$. If $n \equiv$ $0, k+1, k+2, \ldots, 2 k(\bmod (2 k+1))$, then we give values of depth and Stanley depth of the quotient ring of the edge ideal associated to the $k^{t h}$ power of a cycle on $n$ vertices and tight bounds otherwise, in terms of $n$ and $k$. We also compute lower bounds for the Stanley depth of the edge ideals associated to the $k^{t h}$ power of a path and a cycle and prove a conjecture of Herzog for these ideals.
\end{abstract}

\section{Introduction}

Let $K$ be a field and $S:=K\left[x_{1}, \ldots, x_{n}\right]$ the polynomial ring over $K$. Let $M$ be a finitely generated $\mathbb{Z}^{n}$-graded $S$-module. A Stanley decomposition of $M$ is a presentation of the $K$-vector space $M$ as a finite direct sum $\mathcal{D}: M=$ $\oplus_{i=1}^{s} v_{i} K\left[W_{i}\right]$, where $v_{i} \in M, W_{i} \subseteq\left\{x_{1}, \ldots, x_{n}\right\}$, and $v_{i} K\left[W_{i}\right]$ denotes the $K$-subspace of $M$, which is generated by all elements $v_{i} w$, where $w$ is a monomial in $K\left[W_{i}\right]$. The $\mathbb{Z}^{n}$-graded $K$-subspace $v_{i} K\left[W_{i}\right] \subset M$ is called a Stanley space of dimension $\left|W_{i}\right|$, if $v_{i} K\left[W_{i}\right]$ is a free $K\left[W_{i}\right]$-module, where $\left|W_{i}\right|$ denotes the cardinality of $W_{i}$. Define $\operatorname{sdepth}(\mathcal{D})=\min \left\{\left|W_{i}\right|: i=1, \ldots, s\right\}$,

\footnotetext{
Key Words: Monomial ideal, edge ideal, depth, Stanley decomposition, Stanley depth. 2010 Mathematics Subject Classification: Primary 13C15; Secondary 13F20; 05C38;

Received: 06.11.2018

Accepted: 11.01.2019
} $05 \mathrm{E} 99$. 
and $\operatorname{sdepth}(M)=\max \{\operatorname{sdepth}(\mathcal{D}): \mathcal{D}$ is a Stanley decomposition of $M\}$. The number $\operatorname{sdepth}(\mathcal{D})$ is called the Stanley depth of decomposition $\mathcal{D}$ and $\operatorname{sdepth}(M)$ is called the Stanley depth of $M$. Stanley conjectured in [24] that $\operatorname{sdepth}(M) \geq \operatorname{depth}(M)$ for any $\mathbb{Z}^{n}$-graded $S$-module $M$. This conjecture was disproved by Duval et al. in [8] as was expected due to different nature of these two invariants. However, the relation between Stanley depth and some other invariants has already been established; see [11, 12, 21, 26]. In [11], Herzog, Vladoiu and Zheng proved that the Stanley depth of $M$ can be computed in a finite number of steps, if $M=J / I$, where $I \subset J \subset S$ are monomial ideals. But practically it is too hard to compute Stanley depth by using this method; see for instance, $[2,5,15,16]$. For computing Stanley depth for some classes of modules we refer the reader to $[14,20,22,23]$. In this paper we attempt to find values and reasonable bounds for depth and Stanley depth of $I$ and $S / I$, where $I$ is the edge ideal of a power of a path or a cycle. We also compare the values of $\operatorname{sdepth}(I)$ and $\operatorname{sdepth}(S / I)$ and give positive answers to the following conjecture of Herzog.

Conjecture 1.1. [9] Let $I \subset S$ be a monomial ideal then $\operatorname{sdepth}(I) \geq$ $\operatorname{sdepth}(S / I)$.

The above conjecture is proved in some other cases; see [13, 16, 20, 23]. The paper is organized as follows: First two sections are devoted to introduction, definitions, notation, and discussion of some known results. In third section, we compute depth and Stanley depth of $S / I\left(P_{n}^{k}\right)$, where $I\left(P_{n}^{k}\right)$ denotes the edge ideal of the $k^{t h}$ power of a path $P_{n}$ on $n$ vertices. Let for $q \in \mathbb{Q},\lceil q\rceil$ denotes the smallest integer greater than or equal to $q$. Then in Theorems 3.8 and 3.14 we prove that

$$
\operatorname{depth}\left(S / I\left(P_{n}^{k}\right)\right)=\operatorname{sdepth}\left(S / I\left(P_{n}^{k}\right)\right)=\left\lceil\frac{n}{2 k+1}\right\rceil .
$$

Let $I\left(C_{n}^{k}\right)$ be the edge ideal of the $k^{t h}$ power of a cycle $C_{n}$ on $n$ vertices. In fourth section we give some lower bounds for depth and Stanley depth of $S / I\left(C_{n}^{k}\right)$; see Theorems 4.5 and 4.7. If $n \geq 2 k+2$, then by Corollaries 4.6 and 4.8 we prove that if $n \equiv 0, k+1, \ldots, 2 k(\bmod (2 k+1))$ then $\operatorname{depth}\left(S / I\left(C_{n}^{k}\right)\right)=$ $\operatorname{sdepth}\left(S / I\left(C_{n}^{k}\right)\right)=\left\lceil\frac{n}{2 k+1}\right\rceil$. Otherwise,

$$
\left\lceil\frac{n}{2 k+1}\right\rceil-1 \leq \operatorname{depth}\left(S / I\left(C_{n}^{k}\right)\right), \operatorname{sdepth}\left(S / I\left(C_{n}^{k}\right)\right) \leq\left\lceil\frac{n}{2 k+1}\right\rceil .
$$

Last section is devoted to Conjecture 1.1 for $I\left(P_{n}^{k}\right)$ and $I\left(C_{n}^{k}\right)$. By our Theorem 5.2 we have

$$
\operatorname{sdepth}\left(I\left(P_{n}^{k}\right)\right) \geq\left\lceil\frac{n}{2 k+1}\right\rceil+1,
$$


which shows that $I\left(P_{n}^{k}\right)$ satisfies Conjecture 1.1. Let $n \geq 2 k+1$. Proposition 5.3 gives a lower bound for $I\left(C_{n}^{k}\right) / I\left(P_{n}^{k}\right)$ that is

$$
\operatorname{sdepth}\left(I\left(C_{n}^{k}\right) / I\left(P_{n}^{k}\right)\right) \geq\left\lceil\frac{n+k+1}{2 k+1}\right\rceil .
$$

Corollary 5.5 of this paper proves that $I\left(C_{n}^{k}\right)$ satisfies Conjecture 1.1.

\section{Definitions and notation}

Throughout this paper $\mathfrak{m}$ denotes the unique maximal graded ideal $\left(x_{1}, \ldots, x_{n}\right)$ of $S$. We set $S_{m}:=K\left[x_{1}, x_{2}, \ldots, x_{m}\right], \operatorname{supp}(v):=\left\{i: x_{i} \mid v\right\}$ and $\operatorname{supp}(I):=$ $\left\{i: x_{i} \mid u\right.$, for some $\left.u \in \mathcal{G}(I)\right\}$, where $\mathcal{G}(I)$ denotes the unique minimal set of monomial generators of the monomial ideal $I$. Let $I \subset S$ be an ideal. Then we write $I$ instead of $I S$. Thus every ideal will be considered an ideal of $S$ unless otherwise stated. Let $I$ and $J$ be monomial ideals of $S$, then for $I+J$ we write $(I, J)$.

We review some notation and refer the reader to [3] for further details. Let $G$ be a simple graph. For a positive integer $k$, the $k^{t h}$ power of graph $G$ is another graph $G^{k}$ on the same set of vertices, such that two vertices are adjacent in $G^{k}$ when their distance in $G$ is at most $k$. In the whole paper we label the vertices of the graph $G$ by $1,2, \ldots, n$. We denote the set of vertices of $\mathrm{G}$ by $[n]:=\{1,2, \ldots, n\}$ and its edge set by $E(G)$. We assume that all graphs and their powers are simple graphs. We also assume that all graphs have at least two vertices and a non-empty edge set. For a graph $G$, the edge ideal $I(G)$ associated to $G$ is defined as $I(G):=\left(x_{i} x_{j}:\{i, j\} \in E(G)\right)$. For $n \geq 2$, a graph $G$ is called a path if $E(G)=\{\{i, i+1\}: i \in[n-1]\}$. A path on $n$ vertices is denoted by $P_{n}$. For $n \geq 3$, a graph $G$ is called a cycle if $E(G)=\{\{i, i+1\}: i \in[n-1]\} \cup\{1, n\}$. A cycle on $n$ vertices is denoted by $C_{n}$. For $n \geq 2$, the $k^{t h}$ power of a path, denoted by $P_{n}^{k}$, is a graph such that for all $1 \leq i<j \leq n,\{i, j\} \in E\left(P_{n}^{k}\right)$ if and only if $0<j-i \leq k$. If $n \leq k+1$, then $P_{n}^{k}$ is a complete graph on $n$ vertices. If $n \geq k+2$, then

$$
\begin{aligned}
& E\left(P_{n}^{k}\right)=\cup_{i=1}^{n-k}\{\{i, i+1\},\{i, i+2\}, \ldots,\{i, i+k\}\} \cup \\
& \cup_{j=n-k+1}^{n-1}\{\{j, j+1\},\{j, j+2\}, \ldots,\{j, n\}\} .
\end{aligned}
$$

For $n \geq 3$, the $k^{\text {th }}$ power of a cycle, denoted by $C_{n}^{k}$, is a graph such that for all vertices $1 \leq i, j \leq n,\{i, j\} \in E\left(C_{n}^{k}\right)$ if and only if $|j-i| \leq k$ or $|j-i| \geq n-k$. If $n \leq 2 k+1$, then $C_{n}^{k}$ is a complete graph on $n$ vertices. If $n \geq 2 k+2$, then

$$
E\left(C_{n}^{k}\right)=E\left(P_{n}^{k}\right) \cup \cup_{l=1}^{k}\{\{l, l+n-k\},\{l, l+n-k+1\},\{l, l+n-k+2\}, \ldots,\{l, n\}\} .
$$


For examples of powers of paths and cycles see Figures 1 and 2 .

If $n \leq k+1$, then $I\left(P_{n}^{k}\right)$ is a squarefree Veronese ideal of degree 2. If $n \geq k+2$, then

$$
\begin{aligned}
\mathcal{G}\left(I\left(P_{n}^{k}\right)\right)= & \cup_{i=1}^{n-k}\left\{x_{i} x_{i+1}, x_{i} x_{i+2}, \ldots, x_{i} x_{i+k}\right\} \cup \\
& \cup_{j=n-k+1}^{n-1}\left\{x_{j} x_{j+1}, x_{j} x_{j+2}, \ldots, x_{j} x_{n}\right\} .
\end{aligned}
$$

If $n \leq 2 k+1$, then $I\left(C_{n}^{k}\right)$ is a squarefree Veronese ideal of degree 2. If $n \geq 2 k+2$, then

$$
\mathcal{G}\left(I\left(C_{n}^{k}\right)\right)=\mathcal{G}\left(I\left(P_{n}^{k}\right)\right) \cup \cup_{l=1}^{k}\left\{x_{l} x_{l+n-k}, x_{l} x_{l+n-k+1}, \ldots, x_{l} x_{n}\right\} .
$$

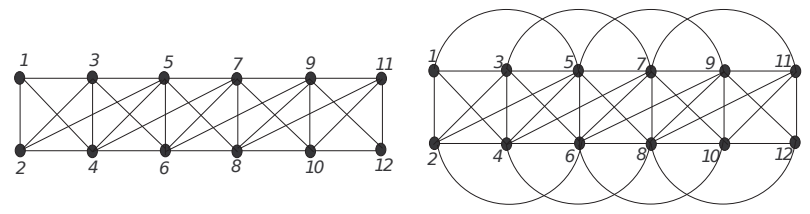

Figure 1: From left to right, $P_{12}^{3}$ and $P_{12}^{4}$ respectively.
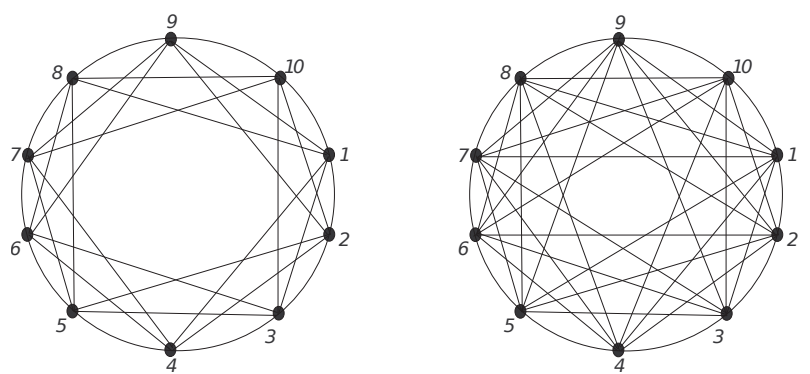

Figure 2: From left to right, $C_{10}^{3}$ and $C_{10}^{4}$ respectively.

Lemma 2.1 ([17, Lemma 3]). If $n \geq k+1$, then $\left|\mathcal{G}\left(I\left(P_{n}^{k}\right)\right)\right|=n k-\frac{k(k+1)}{2}$.

Remark 2.2. If $n \geq 2 k+1$, then $\left|\mathcal{G}\left(I\left(C_{n}^{k}\right)\right)\right|=n k$.

Let $G$ be a graph and $i \in[n]$, then $N_{G}\left(x_{i}\right):=\left\{x_{j}: x_{i} x_{j} \in \mathcal{G}(I(G))\right\}$, where $j \in[n] \backslash\{i\}$. For $k \geq 2,0 \leq i \leq k-1$ and $n \geq 2 k+2$, let $A_{n-k-1}$, 
$A_{n-k+i}, B_{n-k+i}$ and $D_{n-k+i}$ denote the monomial prime ideals of $S$, such that $A_{n-k-1}=(0), A_{n-k+i}:=\left(x_{n-k}, x_{n-k+1}, \ldots, x_{n-k+i}\right)$,

$$
\begin{aligned}
B_{n-k+i}:=\left(x_{j}:\right. & \left.x_{j} \in N_{P_{n}^{k}}\left(x_{n-k+i}\right)\right) \\
& =\left(x_{n-2 k+i}, x_{n-2 k+i+1}, \ldots, x_{n-k+i-1}, x_{n-k+i+1}, \ldots, x_{n}\right),
\end{aligned}
$$

and $D_{n-k+i}:=\left(x_{j}: x_{j} \in N_{C_{n}^{k}}\left(x_{n-k+i}\right)\right)$. Thus if $i=0$, then

$$
D_{n-k+i}=\left(x_{n-2 k}, x_{n-2 k+1}, \ldots, x_{n-k-1}, x_{n-k+1}, \ldots, x_{n}\right)
$$

and if $1 \leq i \leq k-1$, then

$$
D_{n-k+i}=\left(x_{n-2 k+i}, x_{n-2 k+i+1}, \ldots, x_{n-k+i-1}, x_{n-k+i+1}, \ldots, x_{n}, x_{1}, \ldots, x_{i}\right) .
$$

These monomial prime ideals and the following function play important role in the proof of our main theorems. For $k \geq 2$ and $2 k+2 \leq n \leq 3 k+1$, we define a function

$$
\begin{gathered}
f:\{n-k, n-k+1, \ldots, n-k+i, \ldots, n-1\} \longrightarrow \mathbb{Z}^{+} \cup\{0\}, \text { by } \\
f(n-k+i)= \begin{cases}k, & \text { if } n-2 k-1+i \geq k+1 ; \\
n-2 k-2+i, & \text { if } 2 \leq n-2 k-1+i<k+1 .\end{cases}
\end{gathered}
$$

In the following we recall some known results that we refer several times in this paper.

Lemma 2.3 (Depth Lemma). If $0 \longrightarrow U \longrightarrow M \longrightarrow N \longrightarrow 0$ is a short exact sequence of modules over a local ring $S$, or a Noetherian graded ring with $S_{0}$ local, then

1. $\operatorname{depth} M \geq \min \{\operatorname{depth} N$, depth $U\}$.

2. $\operatorname{depth} U \geq \min \{\operatorname{depth} M$, $\operatorname{depth} N+1\}$.

3. $\operatorname{depth} N \geq \min \{\operatorname{depth} U-1$, $\operatorname{depth} M\}$.

Lemma 2.4 ([23, Lemma 2.2]). Let $0 \longrightarrow U \longrightarrow V \longrightarrow W \longrightarrow 0$ be a short exact sequence of $\mathbb{Z}^{n}$-graded $S$-modules. Then

$$
\operatorname{sdepth}(V) \geq \min \{\operatorname{sdepth}(U), \operatorname{sdepth}(W)\} .
$$

Lemma 2.5 ([13, Lemma 3.3]). Let $I \subset S$ be a squarefree monomial ideal with $\operatorname{supp}(I)=[n]$ and $v:=x_{i_{1}} x_{i_{2}} \cdots x_{i_{q}} \in S / I$ such that $x_{j} v \in I$ for all $j \in[n] \backslash \operatorname{supp}(v)$. Then $\operatorname{sdepth}(S / I) \leq q$.

The above Lemma can also be seen as an immediate consequence of the result of J. Apel [1, Sec.3]. 


\section{Depth and Stanley of cyclic modules associated to the edge ideals of the powers of a path}

We start this section with some results. These results are essential for computations of depth and Stanley depth of $S / I\left(P_{n}^{k}\right)$.

Lemma 3.1. Let $a \geq 2$ be an integer, $\left\{E_{i}: 1 \leq i \leq a\right\}$ and $\left\{G_{i}: 0 \leq i \leq a\right\}$ be some families of $\mathbb{Z}^{n}$-graded $S$-modules such that we have the following short exact sequences:

$$
\begin{gathered}
0 \longrightarrow E_{1} \longrightarrow G_{0} \longrightarrow G_{1} \longrightarrow 0 \\
0 \longrightarrow E_{2} \longrightarrow G_{1} \longrightarrow G_{2} \longrightarrow 0 \\
\vdots \\
0 \longrightarrow E_{a-1} \longrightarrow G_{a-2} \longrightarrow G_{a-1} \longrightarrow 0 \\
0 \longrightarrow E_{a} \longrightarrow G_{a-1} \longrightarrow G_{a} \longrightarrow 0
\end{gathered}
$$

and $\operatorname{depth}\left(G_{a}\right) \geq \operatorname{depth}\left(E_{a}\right), \operatorname{depth}\left(E_{i}\right) \geq \operatorname{depth}\left(E_{i-1}\right)$ for all $2 \leq i \leq a$. Then $\operatorname{depth}\left(G_{0}\right)=\operatorname{depth}\left(E_{1}\right)$.

Proof. By assumption, we have $\operatorname{depth}\left(G_{a}\right) \geq \operatorname{depth}\left(E_{a}\right)$, applying Depth Lemma on the exact sequence $(a)$ we get $\operatorname{depth}\left(G_{a-1}\right)=\operatorname{depth}\left(E_{a}\right)$. We also have by assumption

$$
\operatorname{depth}\left(G_{a-1}\right)=\operatorname{depth}\left(E_{a}\right) \geq \operatorname{depth}\left(E_{a-1}\right) .
$$

By applying Depth Lemma on the exact sequence $(a-1)$ we have $\operatorname{depth}\left(G_{a-2}\right)=$ $\operatorname{depth}\left(E_{a-1}\right)$. We repeat the same steps on all exact sequences one by one from bottom to top and we get $\operatorname{depth}\left(G_{i-1}\right)=\operatorname{depth}\left(E_{i}\right)$ for all $i$. Thus if $i=1$ then we have $\operatorname{depth}\left(G_{0}\right)=\operatorname{depth}\left(E_{1}\right)$.

Lemma 3.2. Let $k \geq 2$ and $n \geq 2 k+2$. Then

$$
S /\left(I\left(P_{n}^{k}\right), A_{n-1}\right) \cong S_{n-k-1} / I\left(P_{n-k-1}^{k}\right)\left[x_{n}\right] .
$$

Proof. Since

$$
\begin{aligned}
\mathcal{G}\left(I\left(P_{n}^{k}\right)\right)= & \cup_{i=1}^{n-k}\left\{x_{i} x_{i+1}, x_{i} x_{i+2}, \ldots, x_{i} x_{i+k}\right\} \cup \\
& \cup_{i=n-k+1}^{n-1}\left\{x_{i} x_{i+1}, x_{i} x_{i+2}, \ldots, x_{i} x_{n}\right\},
\end{aligned}
$$


so we have

$$
\begin{aligned}
& I\left(P_{n}^{k}\right)+A_{n-1}=A_{n-1}+ \\
& {\left[\sum_{i=1}^{n-2 k-1}\left(x_{i} x_{i+1}, x_{i} x_{i+2}, \ldots, x_{i} x_{i+k}\right)+\sum_{i=n-2 k}^{n-k}\left(x_{i} x_{i+1}, x_{i} x_{i+2}, \ldots, x_{i} x_{i+k}\right)+\right.} \\
& \left.\sum_{i=n-k+1}^{n-1}\left(x_{i} x_{i+1}, x_{i} x_{i+2}, \ldots, x_{i} x_{n}\right)\right]=\sum_{i=1}^{n-2 k-1}\left(x_{i} x_{i+1}, x_{i} x_{i+2}, \ldots, x_{i} x_{i+k}\right)+ \\
& \sum_{i=n-2 k}^{n-k-2}\left(x_{i} x_{i+1}, x_{i} x_{i+2}, \ldots, x_{i} x_{n-k-1}\right)+A_{n-1}=I\left(P_{n-k-1}^{k}\right)+A_{n-1} .
\end{aligned}
$$

Thus the required result follows.

Lemma 3.3. Let $k \geq 2,0 \leq i \leq k-1$ and $n \geq 3 k+2$. Then

$$
S /\left(I\left(P_{n}^{k}\right): x_{n-k+i}\right) \cong S_{n-2 k-1+i} / I\left(P_{n-2 k-1+i}^{k}\right)\left[x_{n-k+i}\right] .
$$

Proof. It is enough to prove that $\left(I\left(P_{n}^{k}\right): x_{n-k+i}\right)=\left(I\left(P_{n-2 k-1+i}^{k}\right), B_{n-k+i}\right)$. Clearly

$$
I\left(P_{n-2 k-1+i}^{k}\right) \subset I\left(P_{n}^{k}\right) \subset\left(I\left(P_{n}^{k}\right): x_{n-k+i}\right) .
$$

Let $u \in B_{n-k+i}$, then by definition of $I\left(P_{n}^{k}\right), u x_{n-k+i} \in I\left(P_{n}^{k}\right)$ that is $u \in\left(I\left(P_{n}^{k}\right): x_{n-k+i}\right)$. Thus $B_{n-k+i} \subset\left(I\left(P_{n}^{k}\right): x_{n-k+i}\right)$ and we have $\left(I\left(P_{n-2 k-1+i}^{k}\right), B_{n-k+i}\right) \subset\left(I\left(P_{n}^{k}\right): x_{n-k+i}\right)$. Now for the other inclusion, let $w$ be a monomial generator of $\left(I\left(P_{n}^{k}\right): x_{n-k+i}\right)$, then $w=\frac{v}{\operatorname{gcd}\left(v, x_{n-k+i}\right)}$, where $v \in \mathcal{G}\left(I\left(P_{n}^{k}\right)\right)$. If $\operatorname{supp}(v) \cap \mathcal{G}\left(B_{n-k+i}\right) \neq \emptyset$, then we have $w \in \mathcal{G}\left(B_{n-k+i}\right)$ and if $\operatorname{supp}(v) \cap \mathcal{G}\left(B_{n-k+i}\right)=\emptyset$, then $w \in \mathcal{G}\left(I\left(P_{n}^{k}\right)\right) \cap K\left[x_{1}, x_{2}, \ldots, x_{n-2 k-1+i}\right]=$ $\mathcal{G}\left(I\left(P_{n-2 k-1+i}^{k}\right)\right)$.

Lemma 3.4. Let $n \geq 3 k+2$ and $0 \leq i \leq k-1$, then we have

$$
S /\left(\left(I\left(P_{n}^{k}\right), A_{n-k+(i-1)}\right): x_{n-k+i}\right) \cong S_{n-2 k-1+i} / I\left(P_{n-2 k-1+i}^{k}\right)\left[x_{n-k+i}\right] .
$$

Proof. As $\left(\left(I\left(P_{n}^{k}\right), A_{n-k+(i-1)}\right): x_{n-k+i}\right)=\left(\left(I\left(P_{n}^{k}\right): x_{n-k+i}\right), A_{n-k+(i-1)}\right)$. Now using the proof of Lemma 3.3 we obtain

$$
\begin{aligned}
& \left(\left(I\left(P_{n}^{k}\right): x_{n-k+i}\right), A_{n-k+(i-1)}\right)= \\
& \quad\left(I\left(P_{n-2 k-1+i}^{k}\right), B_{n-k+i}, A_{n-k+(i-1)}\right)=\left(I\left(P_{n-2 k-1+i}^{k}\right), B_{n-k+i}\right),
\end{aligned}
$$

as $A_{n-k+(i-1)} \subset B_{n-k+i}$. Thus the required result follows by Lemma 3.3. 
Remark 3.5. Let $m \geq 2$ and $I\left(P_{m}^{m-1}\right) \subset S_{m}=K\left[x_{1}, x_{2}, \ldots, x_{m}\right]$ be the edge ideal of the $(m-1)^{t h}$ power of path $P_{m}$. Then $I\left(P_{m}^{m-1}\right)$ is a squarefree Veronese ideal of degree 2 in variables $x_{1}, x_{2}, \ldots, x_{m}$. Thus by [10, Corollary 10.3.7] and Theorem 3.9

$$
\operatorname{depth}\left(S_{m} / I\left(P_{m}^{m-1}\right)\right)=\operatorname{sdepth}\left(S_{m} / I\left(P_{m}^{m-1}\right)\right)=1 .
$$

Remark 3.6. Let $k \geq 2$ and $2 k+2 \leq n \leq 3 k+1$, then it is easy to see that

(1) If $n=2 k+2$, then

$$
S /\left(I\left(P_{n}^{k}\right): x_{n-k}\right)=S /\left(x_{2}, \ldots, x_{n-k-1}, x_{n-k+1}, \ldots, x_{n}\right) \cong K\left[x_{1}, x_{n-k}\right] .
$$

(2) If $0 \leq i \leq k-1$ and $n>2 k+2$, then

$$
\begin{gathered}
S /\left(I\left(P_{n}^{k}\right): x_{n-k+i}\right)=S /\left(\left(I\left(P_{n}^{k}\right), A_{n-k+(i-1)}\right): x_{n-k+i}\right) \\
\cong S_{n-2 k-1+i} / I\left(P_{n-2 k-1+i}^{f(n-k+i)}\right)\left[x_{n-k+i}\right] \\
=\left\{\begin{array}{cc}
S_{n-2 k-1+i} / I\left(P_{n-2 k-1+i}^{k}\right)\left[x_{n-k+i}\right], \quad \text { if } n-2 k-1+i \geq k+1 ; \\
S_{n-2 k-1+i} / I\left(P_{n-2 k-1+i}^{n-2 k-2+i}\right)\left[x_{n-k+i}\right], & \text { otherwise. }
\end{array}\right.
\end{gathered}
$$

We recall a lemma from [11] which is heavily used in this paper.

Lemma 3.7 ([11, Lemma 3.6]). Let $J \subset I$ be monomial ideals of $S$, and let $T=S\left[x_{n+1}\right]$ be the polynomial ring over $S$ in the variable $x_{n+1}$. Then $\operatorname{depth}(I T / J T)=\operatorname{depth}(I / J)+1$ and $\operatorname{sdepth}(I T / J T)=\operatorname{sdepth}(I / J)+1$.

Theorem 3.8. Let $n \geq 2$. Then $\operatorname{depth}\left(S / I\left(P_{n}^{k}\right)\right)=\left\lceil\frac{n}{2 k+1}\right\rceil$.

Proof. (a) If $n \leq k+1$, then $I\left(P_{n}^{k}\right)$ is a squarefree Veronese ideal thus by $\operatorname{Remark} 3.5, \operatorname{depth}\left(S / I\left(P_{n}^{k}\right)\right)=1=\left\lceil\frac{n}{2 k+1}\right\rceil$.

(b) For $n \geq k+2$, we consider the following cases:

(1) If $k=1$, then by $\left[18\right.$, Lemma 2.8] we have $\operatorname{depth}\left(S / I\left(P_{n}^{1}\right)\right)=\left\lceil\frac{n}{3}\right\rceil=$ $\left\lceil\frac{n}{2 k+1}\right\rceil$.

(2) If $k \geq 2$ and $k+2 \leq n \leq 2 k+1$, then we get $\operatorname{depth}\left(S / I\left(P_{n}^{k}\right)\right) \geq 1$ as $\mathfrak{m} \notin \operatorname{Ass}\left(S / I\left(P_{n}^{k}\right)\right)$. Since $x_{k+1} \notin I\left(P_{n}^{k}\right)$ and $x_{s} x_{k+1} \in \mathcal{G}\left(I\left(P_{n}^{k}\right)\right)$ for all $s \in\{1, \ldots k, k+2, \ldots, n\}$, therefore we have $\left(I\left(P_{n}^{k}\right): x_{k+1}\right)=$ $\left(x_{1}, \ldots x_{k}, x_{k+2}, \ldots, x_{n}\right)$. By [23, Corollary 1.3], we have

$$
\begin{aligned}
\operatorname{depth}\left(S / I\left(P_{n}^{k}\right)\right) \leq \operatorname{depth}\left(S /\left(I\left(P_{n}^{k}\right): x_{k+1}\right)\right) & \\
& =\operatorname{depth}\left(S /\left(x_{1}, \ldots x_{k}, x_{k+2}, \ldots, x_{n}\right)\right)=1 .
\end{aligned}
$$

$\operatorname{Thus} \operatorname{depth}\left(S / I\left(P_{n}^{k}\right)\right)=1=\left\lceil\frac{n}{2 k+1}\right\rceil$. 
(3) For $k \geq 2,2 k+2 \leq n \leq 3 k+1$ and $0 \leq i \leq k-1$, consider the family of short exact sequences

$$
\begin{aligned}
& 0 \longrightarrow S /\left(\left(I\left(P_{n}^{k}\right), A_{n-k+(i-1)}\right): x_{n-k+i}\right) \stackrel{\cdot x_{n-k+i}}{\longrightarrow} \\
& \quad S /\left(I\left(P_{n}^{k}\right), A_{n-k+(i-1)}\right) \longrightarrow S /\left(I\left(P_{n}^{k}\right), A_{n-k+i}\right) \longrightarrow 0
\end{aligned}
$$

By Lemma 3.2, $S /\left(I\left(P_{n}^{k}\right), A_{n-1}\right) \cong S_{n-k-1} / I\left(P_{n-k-1}^{k}\right)\left[x_{n}\right]$. Since we are considering the case $2 k+2 \leq n \leq 3 k+1$ which implies that $k+1 \leq n-k-1 \leq 2 k$. If $n-k-1=k+1$ then $S_{n-k-1} / I\left(P_{n-k-1}^{k}\right)=S_{k+1} / I\left(P_{k+1}^{k}\right)$, by Remark 3.5 and Lemma 3.7 we have depth $\left(S /\left(I\left(P_{n}^{k}\right), A_{n-1}\right)\right)=2$. If $k+1<n-k-1 \leq$ $2 k$, then by case(b) $(2) \operatorname{depth}\left(S_{n-k-1} / I\left(P_{n-k-1}^{k}\right)\right)=1$. Thus by Lemma 3.7 we have $\operatorname{depth}\left(S /\left(I\left(P_{n}^{k}\right), A_{n-1}\right)\right)=2$. Now we show that depth $\left(S /\left(I\left(P_{n}^{k}\right): x_{n-k}\right)\right)=2$. For this we consider two cases: If $n=2 k+2$, then by Remark 3.6

$$
\begin{aligned}
& S /\left(I\left(P_{n}^{k}\right): x_{n-k}\right)= \\
& \quad S /\left(x_{2}, x_{3}, \ldots, x_{n-k-1}, x_{n-k+1}, \ldots, x_{n}\right) \cong K\left[x_{1}, x_{n-k}\right],
\end{aligned}
$$

and thus depth $\left(S /\left(I\left(P_{n}^{k}\right): x_{n-k}\right)\right)=2$. If $n>2 k+2$, by Remark 3.6 we have

$$
S /\left(I\left(P_{n}^{k}\right): x_{n-k}\right) \cong S_{n-2 k-1} / I\left(P_{n-2 k-1}^{n-2 k-2}\right)\left[x_{n-k}\right],
$$

where $2 \leq n-2 k-1 \leq k$. Thus by Remark 3.5 and Lemma 3.7 we get $\operatorname{depth}\left(S /\left(I\left(P_{n}^{k}\right): x_{n-k}\right)\right)=2$. Now for $1 \leq i \leq k-1$, by Remark 3.6 we obtain

$$
\begin{aligned}
S /\left(\left(I\left(P_{n}^{k}\right), A_{n-k+(i-1)}\right):\right. & \left.x_{n-k+i}\right)=S /\left(I\left(P_{n}^{k}\right): x_{n-k+i}\right) \\
& \cong S_{n-2 k-1+i} / I\left(P_{n-2 k-1+i}^{f(n-k+i)}\right)\left[x_{n-k+i}\right] .
\end{aligned}
$$

Let $T:=S_{n-2 k-1+i} / I\left(P_{n-2 k-1+i}^{f(n-k+i)}\right)\left[x_{n-k+i}\right]$. We consider the following cases:

(i) If $k+1=n-2 k-1+i$, then $T=S_{k+1} / I\left(P_{k+1}^{k}\right)\left[x_{n-k+i}\right]$, thus by case(a) and Lemma 3.7 we have $\operatorname{depth}(T)=2$.

(ii) For $k+1<n-2 k-1+i, T=S_{n-2 k-1+i} / I\left(P_{n-2 k-1+i}^{k}\right)\left[x_{n-k+i}\right]$. Since $k+2 \leq n-2 k-1+i \leq 2 k-1$, thus by case(b)(2) and Lemma 3.7 we have $\operatorname{depth}(T)=2$. 
(iii) If $2 \leq n-2 k-1+i<k+1$, then

$T=S_{n-2 k-1+i} / I\left(P_{n-2 k-1+i}^{n-2 k-2+i}\right)\left[x_{n-k+i}\right]$, by Remark 3.5 and Lemma 3.7 we have $\operatorname{depth}(T)=2$.

Thus by Lemma 3.1 we have $\operatorname{depth}\left(S / I\left(P_{n}^{k}\right)\right)=2$.

(4) For $k \geq 2, n \geq 3 k+2$ and $0 \leq i \leq k-1$, consider the family of short exact sequences

$$
\begin{aligned}
& 0 \longrightarrow S /(\left.\left(I\left(P_{n}^{k}\right), A_{n-k+(i-1)}\right): x_{n-k+i}\right) \stackrel{\cdot x_{n-k+i}}{\longrightarrow} \\
& S /\left(I\left(P_{n}^{k}\right), A_{n-k+(i-1)}\right) \longrightarrow S /\left(I\left(P_{n}^{k}\right), A_{n-k+i}\right) \longrightarrow 0
\end{aligned}
$$

By Lemma 3.2, $\left.S /\left(I\left(P_{n}^{k}\right), A_{n-1}\right)\right) \cong S_{n-k-1} / I\left(P_{n-k-1}^{k}\right)\left[x_{n}\right]$. Thus by induction on $n$ and Lemma 3.7 we have $\operatorname{depth}\left(S /\left(I\left(P_{n}^{k}\right), A_{n-1}\right)\right)=$ $\left\lceil\frac{n-k-1}{2 k+1}\right\rceil+1$. By Lemma 3.4 we have

$S /\left(\left(I\left(P_{n}^{k}\right), A_{n-k+(i-1)}\right): x_{n-k+i}\right) \cong S_{n-2 k-1+i} / I\left(P_{n-2 k-1+i}^{k}\right)\left[x_{n-k+i}\right]$.

Thus by induction on $n$ and Lemma 3.7 we have

$$
\operatorname{depth}\left(S /\left(\left(I\left(P_{n}^{k}\right), A_{n-k+(i-1)}\right): x_{n-k+i}\right)\right)=\left\lceil\frac{n-2 k-1+i}{2 k+1}\right\rceil+1 .
$$

Here we can see that

$$
\begin{aligned}
& \operatorname{depth}(S /(\left.\left.I\left(P_{n}^{k}\right), A_{n-1}\right)\right)=\left\lceil\frac{n-k-1}{2 k+1}\right\rceil+1 \geq \\
&\left.\left\lceil\frac{n-k-2}{2 k+1}\right\rceil+1=\operatorname{depth}\left(S /\left(I\left(P_{n}^{k}\right), A_{n-2}\right): x_{n-1}\right)\right),
\end{aligned}
$$

and for all $1 \leq i \leq k-1$,

$$
\begin{gathered}
\operatorname{depth}\left(S /\left(\left(I\left(P_{n}^{k}\right), A_{n-k+(i-1)}\right): x_{n-k+i}\right)\right)=\left\lceil\frac{n-2 k-1+i}{2 k+1}\right\rceil+1 \geq \\
\left\lceil\frac{n-2 k-2+i}{2 k+1}\right\rceil+1=\operatorname{depth}\left(S /\left(\left(I\left(P_{n}^{k}\right), A_{n-k+(i-2)}\right): x_{n-k+(i-1)}\right)\right) .
\end{gathered}
$$

Thus by Lemma 3.1 we have $\operatorname{depth}\left(S / I\left(P_{n}^{k}\right)\right)=\left\lceil\frac{n-2 k-1}{2 k+1}\right\rceil+1=$ $\left\lceil\frac{n}{2 k+1}\right\rceil$.

Let $d \in[n]$ and $I_{n, d}:=(u \in S$ square free monomial : $\operatorname{deg}(u)=d)$. Then $I_{n, d}$ is called squarefree Veronese ideal of degree $d$ in the variables $x_{1}, x_{2}, \ldots, x_{n}$. Cimpoeas proved the following theorems: 
Theorem 3.9 ([5, Theorem 1.1]). (1) $\operatorname{sdepth}\left(S / I_{n, d}\right)=d-1$.

(2) $d \leq \operatorname{sdepth}\left(I_{n, d}\right) \leq \frac{n-d}{d+1}+d$.

Theorem 3.10 ([7, Theorem 1.4]). Let $M$ be a $\mathbb{Z}^{n}$-graded $S$-module. If $\operatorname{sdepth}(M)=0$, then $\operatorname{depth}(M)=0$. Conversely, if $\operatorname{depth}(M)=0$ and $\operatorname{dim}_{K}\left(M_{a}\right)=1$ for any $a \in \mathbb{Z}^{n}$, then $\operatorname{sdepth}(M)=0$.

Lemma 3.11 ([25, Lemma 4]). Let $n \geq 2$, then $\operatorname{sdepth}\left(S / I\left(P_{n}^{1}\right)\right)=\left\lceil\frac{n}{3}\right\rceil$.

Example 3.12. Let $n \geq 2$, and $n \leq 2 k+1$, then $\operatorname{sdepth}\left(S / I\left(P_{n}^{k}\right)\right)=1$.

Proof. If $n \leq k+1$, then by Theorem $3.9 \operatorname{sdepth}\left(S / I\left(P_{n}^{k}\right)\right)=1$. Now if $k+2 \leq$ $n \leq 2 k+1$, then $\operatorname{depth}\left(S / I\left(P_{n}^{k}\right)\right) \geq 1$ as $\mathfrak{m} \notin \operatorname{Ass}\left(S / I\left(P_{n}^{k}\right)\right)$, thus by Theorem $3.10 \operatorname{sdepth}\left(S / I\left(P_{n}^{k}\right)\right) \geq 1$. Since $x_{k+1} \notin I\left(P_{n}^{k}\right)$ and $x_{i} x_{k+1} \in \mathcal{G}\left(I\left(P_{n}^{k}\right)\right)$ for all $i \in\{1, \ldots k, k+2, \ldots, n\}$, therefore $\left(I\left(P_{n}^{k}\right): x_{k+1}\right)=\left(x_{1}, \ldots x_{k}, x_{k+2}, \ldots, x_{n}\right)$. Thus by $\left[4, \operatorname{Proposition~2.7]} \operatorname{sdepth}\left(S / I\left(P_{n}^{k}\right)\right) \leq \operatorname{sdepth}\left(S /\left(I\left(P_{n}^{k}\right): x_{k+1}\right)\right)=\right.$ $\operatorname{sdepth}\left(S /\left(x_{1}, \ldots x_{k}, x_{k+2}, \ldots, x_{n}\right)\right)=1$.

Proposition 3.13. Let $k \geq 2$ and $n \geq 2 k+2$. Then

$$
\operatorname{sdepth}\left(S / I\left(P_{n}^{k}\right)\right) \geq\left\lceil\frac{n}{2 k+1}\right\rceil .
$$

Proof. (1) If $2 k+2 \leq n \leq 3 k+1$, then by applying Lemma 2.4 on the exact sequences in case(b)(3) of Theorem 3.8 we get $\operatorname{sdepth}\left(S / I\left(P_{n}^{k}\right)\right) \geq 2=$ $\left\lceil\frac{n}{2 k+1}\right\rceil$.

(2) If $n \geq 3 k+2$, then the proof is similar to Theorem 3.8. We apply Lemma 2.4 on the exact sequences in case(b)(4) of Theorem 3.8 and obtain

$$
\begin{aligned}
& \operatorname{sdepth}\left(S / I\left(P_{n}^{k}\right)\right) \geq \min \left\{\operatorname{sdepth}\left(S /\left(I\left(P_{n}^{k}\right), A_{n-1}\right)\right),\right. \\
& \left.\quad \min _{i=0}^{k-1}\left\{\operatorname{sdepth}\left(S /\left(\left(I\left(P_{n}^{k}\right), A_{n-k+(i-1)}\right): x_{n-k+i}\right)\right)\right\}\right\} \geq\left\lceil\frac{n}{2 k+1}\right\rceil .
\end{aligned}
$$

Theorem 3.14. Let $n \geq 2$, then $\operatorname{sdepth}\left(S / I\left(P_{n}^{k}\right)\right)=\left\lceil\frac{n}{2 k+1}\right\rceil$.

Proof. If $k=1$, then the result follows by Lemma 3.11. Let $k \geq 2$. If $n \leq 2 k+1$, then by Example 3.12 we have the required result. If $n \geq 2 k+2$, then by Proposition 3.13 we have

$$
\operatorname{sdepth}\left(S / I\left(P_{n}^{k}\right)\right) \geq\left\lceil\frac{n}{2 k+1}\right\rceil .
$$

We need to prove that $\operatorname{sdepth}\left(S / I\left(P_{n}^{k}\right)\right) \leq\left\lceil\frac{n}{2 k+1}\right\rceil$, for this we consider the following three cases: 
(1) If $n=(2 k+1) l$, where $l \geq 1$. We see that

$$
v=x_{k+1} x_{3 k+2} x_{5 k+3} \cdots x_{(2 k+1) l-k} \in S \backslash I\left(P_{n}^{k}\right),
$$

but $x_{t_{1}} v \in I\left(P_{n}^{k}\right)$ for all $t_{1} \in[n] \backslash \operatorname{supp}(v)$, thus by Lemma 2.5,

$$
\operatorname{sdepth}\left(S / I\left(P_{n}^{k}\right)\right) \leq l=\left\lceil\frac{n}{2 k+1}\right\rceil .
$$

(2) If $n=(2 k+1) l+r$, where $r \in\{1,2,3, \ldots, k+1\}$ and $l \geq 1$, then we have

$$
v=x_{k+1} x_{3 k+2} x_{5 k+3} \cdots x_{(2 k+1) l-k} x_{(2 k+1) l+r} \in S \backslash I\left(P_{n}^{k}\right),
$$

and $x_{t_{2}} v \in I\left(P_{n}^{k}\right)$ for all $t_{2} \in[n] \backslash \operatorname{supp}(v)$, so by Lemma 2.5,

$$
\operatorname{sdepth}\left(S / I\left(P_{n}^{k}\right)\right) \leq l+1=\left\lceil\frac{n}{2 k+1}\right\rceil .
$$

(3) If $n=(2 k+1) l+s$, where $s \in\{k+2, k+3, \ldots, 2 k\}$ and $l \geq 1$, since

$$
v=x_{k+1} x_{3 k+2} x_{5 k+3} \cdots x_{(2 k+1) l+k+1} \in S \backslash I\left(P_{n}^{k}\right),
$$

but $x_{t_{3}} v \in I\left(P_{n}^{k}\right)$ for all $t_{3} \in[n] \backslash \operatorname{supp}(v)$, by Lemma 2.5, we get

$$
\operatorname{sdepth}\left(S / I\left(P_{n}^{k}\right)\right) \leq l+1=\left\lceil\frac{n}{2 k+1}\right\rceil .
$$

\section{Depth and Stanley depth of cyclic modules associated to the edge ideals of the powers of a cycle}

In this section, we compute bounds for depth and Stanley depth of cyclic modules associated to the edge ideals of powers of a cycle. In order to complete the main task of this section we prove the following three lemmas.

Lemma 4.1. Let $k \geq 2$ and $n \geq 3 k+2$, then $S /\left(I\left(C_{n}^{k}\right), A_{n-1}\right) \cong S_{n-k} / I\left(P_{n-k}^{k}\right)$.

Proof. Since $\mathcal{G}\left(I\left(C_{n}^{k}\right)\right)=\mathcal{G}\left(I\left(P_{n}^{k}\right)\right) \cup \cup_{l=1}^{k-1}\left\{x_{l} x_{l+n-k}, x_{l} x_{l+n-k+1}, \ldots, x_{l} x_{n-1}\right\} \cup$ $\left\{x_{1} x_{n}, x_{2} x_{n}, \ldots, x_{k} x_{n}\right\}$, we have

$$
\begin{aligned}
& I\left(C_{n}^{k}\right)+A_{n-1}= \\
& I\left(P_{n}^{k}\right)+\sum_{l=1}^{k-1}\left(x_{l} x_{l+n-k}, x_{l} x_{l+n-k+1}, \ldots, x_{l} x_{n-1}\right)+\left(x_{1} x_{n}, x_{2} x_{n}, \ldots, x_{k} x_{n}\right)+A_{n-1} .
\end{aligned}
$$


Thus by the proof of Lemma 3.2, we obtain $I\left(P_{n}^{k}\right)+A_{n-1}=I\left(P_{n-k-1}^{k}\right)+A_{n-1}$. As

$$
\sum_{l=1}^{k-1}\left(x_{l} x_{l+n-k}, x_{l} x_{l+n-k+1}, \ldots, x_{l} x_{n-1}\right)+A_{n-1}=A_{n-1} .
$$

Therefore $S /\left(I\left(C_{n}^{k}\right), A_{n-1}\right)=S /\left(I\left(P_{n-k-1}^{k}\right), A_{n-1},\left(x_{1} x_{n}, x_{2} x_{n}, \ldots, x_{k} x_{n}\right)\right)$

$$
\cong K\left[x_{1}, x_{2}, \ldots, x_{n-k-1}, x_{n}\right] /\left(I\left(P_{n-k-1}^{k}\right),\left(x_{1} x_{n}, x_{2} x_{n}, \ldots, x_{k} x_{n}\right)\right) \text {. }
$$

After renumbering the variables, we have

$K\left[x_{1}, \ldots, x_{n-k-1}, x_{n}\right] /\left(I\left(P_{n-k-1}^{k}\right),\left(x_{1} x_{n}, x_{2} x_{n}, \ldots, x_{k} x_{n}\right)\right) \cong S_{n-k} / I\left(P_{n-k}^{k}\right)$.

Lemma 4.2. Let $k \geq 2$ and $n \geq 3 k+2$ and $0 \leq i \leq k-1$, then

$$
S /\left(I\left(C_{n}^{k}\right): x_{n-k+i}\right) \cong S_{n-2 k-1} / I\left(P_{n-2 k-1}^{k}\right)\left[x_{n-k+i}\right]
$$

Proof. Let $w$ be a monomial generator of $\left(I\left(C_{n}^{k}\right): x_{n-k+i}\right)$. Then $w=$ $\frac{v}{g c d\left(v, x_{n-k+i}\right)}$, where $v \in \mathcal{G}\left(I\left(C_{n}^{k}\right)\right)$. If $\operatorname{supp}(v) \cap \mathcal{G}\left(D_{n-k+i}\right) \neq \emptyset$, then we have $w \in \mathcal{G}\left(D_{n-k+i}\right)$ and if $\operatorname{supp}(v) \cap \mathcal{G}\left(D_{n-k+i}\right)=\emptyset$ then $w \in E:=\mathcal{G}\left(I\left(C_{n}^{k}\right)\right) \cap$ $K\left[x_{i+1}, x_{i+2}, \ldots, x_{n-2 k-1+i}\right]$. So we obtain $\left(I\left(C_{n}^{k}\right): x_{n-k+i}\right) \subset E+D_{n-k+i}$. The other inclusion being trivial we get $\left(I\left(C_{n}^{k}\right): x_{n-k+i}\right)=E+D_{n-k+i}$, which further implies that $S /\left(I\left(C_{n}^{k}\right): x_{n-k+i}\right)=S /\left(E+D_{n-k+i}\right)$. After renumbering the variables, we have

$$
S /\left(I\left(C_{n}^{k}\right): x_{n-k+i}\right)=S /\left(E, D_{n-k+i}\right) \cong S_{n-2 k-1} / I\left(P_{n-2 k-1}^{k}\right)\left[x_{n-k+i}\right] .
$$

Lemma 4.3. Let $k \geq 2, n \geq 3 k+2$ and $0 \leq i \leq k-1$. Then

$$
S /\left(\left(I\left(C_{n}^{k}\right), A_{n-k+(i-1)}\right): x_{n-k+i}\right) \cong S_{n-2 k-1} / I\left(P_{n-2 k-1}^{k}\right)\left[x_{n-k+i}\right] .
$$

Proof. As $\left(\left(I\left(C_{n}^{k}\right), A_{n-k+(i-1)}\right): x_{n-k+i}\right)=\left(\left(I\left(C_{n}^{k}\right): x_{n-k+i}\right), A_{n-k+(i-1)}\right)$. By using the same arguments as in the proof of Lemma 4.2 we have

$$
\left(\left(I\left(C_{n}^{k}\right): x_{n-k+i}\right), A_{n-k+(i-1)}\right)=\left(E, D_{n-k+i}, A_{n-k+(i-1)}\right)=\left(E, D_{n-k+i}\right)
$$

as $A_{n-k+(i-1)} \subset D_{n-k+i}$. Thus the required result follows by Lemma 4.2.

Corollary 4.4 ([10, Corollary 10.3.7]). Let $2 \leq d<n$. Then

$$
\operatorname{depth}\left(S / I_{n, d}^{t}\right)=\max \{0, n-t(n-d)-1\} .
$$


Theorem 4.5. Let $n \geq 3$, then

$$
\begin{aligned}
\operatorname{depth}\left(S / I\left(C_{n}^{k}\right)\right) & =1, \quad \text { if } n \leq 2 k+1 \\
\operatorname{depth}\left(S / I\left(C_{n}^{k}\right)\right) & \geq\left\lceil\frac{n-k}{2 k+1}\right\rceil, \quad \text { if } n \geq 2 k+2 .
\end{aligned}
$$

Proof. (a) If $n \leq 2 k+1$, then $I\left(C_{n}^{k}\right)$ is a squarefree Veronese ideal of degree 2. Thus by Corollary $4.4, \operatorname{depth}\left(S / I\left(C_{n}^{k}\right)\right)=1$.

(b) For $n \geq 2 k+2$, we consider the following cases:

(1) If $k=1$, then by [6, Proposition 1.3] $\operatorname{depth}\left(S / I\left(C_{n}^{1}\right)\right)=\left\lceil\frac{n-1}{3}\right\rceil$.

(2) If $k \geq 2$ and $2 k+2 \leq n \leq 3 k+1$, then we have $\operatorname{depth}\left(S / I\left(C_{n}^{k}\right)\right) \geq$ $1=\left\lceil\frac{n-k}{2 k+1}\right\rceil$ as $\mathfrak{m} \notin \operatorname{Ass}\left(S / I\left(C_{n}^{k}\right)\right)$.

(3) For $k \geq 2, n \geq 3 k+2$ and $0 \leq i \leq k-1$, consider the family of short exact sequences

$$
\begin{aligned}
& 0 \longrightarrow S /\left(\left(I\left(C_{n}^{k}\right), A_{n-k+(i-1)}\right): x_{n-k+i}\right) \stackrel{x_{n-k+i}}{\longrightarrow} \\
& S /\left(I\left(C_{n}^{k}\right), A_{n-k+(i-1)}\right) \longrightarrow S /\left(I\left(C_{n}^{k}\right), A_{n-k+i}\right) \longrightarrow 0
\end{aligned}
$$

By Lemma 4.1 we have $\left.S /\left(I\left(C_{n}^{k}\right), A_{n-1}\right)\right) \cong S_{n-k} / I\left(P_{n-k}^{k}\right)$. Now by Lemma 4.3, we get

$$
S /\left(\left(I\left(C_{n}^{k}\right), A_{n-k+(i-1)}\right): x_{n-k+i}\right) \cong S_{n-2 k-1} / I\left(P_{n-2 k-1}^{k}\right)\left[x_{n-k+i}\right] .
$$

By Theorem 3.8 and Lemma 3.7, we obtain

$\operatorname{depth}\left(S /\left(\left(I\left(C_{n}^{k}\right), A_{n-k+(i-1)}\right): x_{n-k+i}\right)\right)=\left\lceil\frac{n-2 k-1}{2 k+1}\right\rceil+1=\left\lceil\frac{n}{2 k+1}\right\rceil$.

Again by Theorem 3.8, we have $\operatorname{depth}\left(S /\left(I\left(C_{n}^{k}\right), A_{n-1}\right)\right)=\left\lceil\frac{n-k}{2 k+1}\right\rceil$. Thus by applying Lemma 2.3(1) on the family of short exact sequences we get $\operatorname{depth}\left(S / I\left(C_{n}^{k}\right)\right) \geq\left\lceil\frac{n-k}{2 k+1}\right\rceil$.

Corollary 4.6. Let $n \geq 3$, If $n \geq 2 k+2$, then

$$
\begin{gathered}
\operatorname{depth}\left(S / I\left(C_{n}^{k}\right)\right)=\left\lceil\frac{n}{2 k+1}\right\rceil, \quad \text { if } n \equiv 0, k+1, \ldots, 2 k(\bmod (2 k+1)) ; \\
\left\lceil\frac{n}{2 k+1}\right\rceil-1 \leq \operatorname{depth}\left(S / I\left(C_{n}^{k}\right)\right) \leq\left\lceil\frac{n}{2 k+1}\right\rceil, \quad \text { if } n \equiv 1, \ldots, k(\bmod (2 k+1)) .
\end{gathered}
$$

Proof. By Theorem 4.5, it is enough to prove that $\operatorname{depth}\left(S / I\left(C_{n}^{k}\right)\right) \leq\left\lceil\frac{n}{2 k+1}\right\rceil$, for $k \geq 2$ and $n \geq 2 k+2$. Since $x_{n-k} \notin I\left(C_{n}^{k}\right)$, thus by [23, Corollary 1.3] we have $\operatorname{depth}\left(S / I\left(C_{n}^{k}\right)\right) \leq \operatorname{depth}\left(S /\left(I\left(C_{n}^{k}\right): x_{n-k}\right)\right)$. Now we consider two cases: 
(1) Let $2 k+2 \leq n \leq 3 k+1$, then $S /\left(I\left(C_{n}^{k}\right): x_{n-k}\right)=S /\left(I\left(P_{n}^{k}\right): x_{n-k}\right)$ so by the proof of Theorem 3.8 we have $\operatorname{depth}\left(S /\left(I\left(P_{n}^{k}\right): x_{n-k}\right)\right)=2=$ $\left\lceil\frac{n}{2 k+1}\right\rceil$. Therefore

$$
\operatorname{depth}\left(S / I\left(C_{n}^{k}\right)\right) \leq \operatorname{depth}\left(S /\left(I\left(C_{n}^{k}\right): x_{n-k}\right)\right)=2=\left\lceil\frac{n}{2 k+1}\right\rceil .
$$

(2) Let $n \geq 3 k+2$, then by Lemma 4.2 ,

$$
S /\left(I\left(C_{n}^{k}\right): x_{n-k}\right) \cong S_{n-2 k-1} / I\left(P_{n-2 k-1}^{k}\right)\left[x_{n-k}\right] .
$$

By Lemma 3.7 and Theorem 3.8, $\operatorname{depth}\left(S_{n-2 k-1} / I\left(P_{n-2 k-1}^{k}\right)\left[x_{n-k}\right]\right)=$ $\left\lceil\frac{n}{2 k+1}\right\rceil$. Thus $\operatorname{depth}\left(S / I\left(C_{n}^{k}\right)\right) \leq \operatorname{depth}\left(S /\left(I\left(C_{n}^{k}\right): x_{n-k}\right)\right)=\left\lceil\frac{n}{2 k+1}\right\rceil$.

Theorem 4.7. Let $n \geq 3$, then

$$
\begin{array}{ll}
\operatorname{sdepth}\left(S / I\left(C_{n}^{k}\right)\right) & =1, \quad \text { if } n \leq 2 k+1 \\
\operatorname{sdepth}\left(S / I\left(C_{n}^{k}\right)\right) \geq\left\lceil\frac{n-k}{2 k+1}\right\rceil, & \text { if } n \geq 2 k+2 .
\end{array}
$$

Proof. (a) If $n \leq 2 k+1$, then $\operatorname{sdepth}\left(S / I\left(C_{n}^{k}\right)\right)=1$ by Theorem 3.9.

(b) For $n \geq 2 k+2$, consider the following cases:

(1) If $k=1$, then by [6, Proposition 1.8] $\operatorname{sdepth}\left(S / I\left(C_{n}^{1}\right)\right) \geq\left\lceil\frac{n-1}{3}\right\rceil$.

(2) If $k \geq 2$ and $2 k+2 \leq n \leq 3 k+1$, then $\operatorname{depth}\left(S / I\left(C_{n}^{k}\right)\right) \geq 1$ as $\mathfrak{m} \notin \operatorname{Ass}\left(S / I\left(C_{n}^{k}\right)\right)$, thus by Theorem 3.10, $\operatorname{sdepth}\left(S / I\left(C_{n}^{k}\right)\right) \geq 1=$ $\left\lceil\frac{n-k}{2 k+1}\right\rceil$.

(3) For $k \geq 2, n \geq 3 k+2$ and $0 \leq i \leq k-1$, consider the family of short exact sequences

$$
\begin{aligned}
& 0 \longrightarrow S /\left(\left(I\left(C_{n}^{k}\right), A_{n-k+(i-1)}\right): x_{n-k+i}\right) \stackrel{\cdot x_{n-k+i}}{\longrightarrow} \\
& S /\left(I\left(C_{n}^{k}\right), A_{n-k+(i-1)}\right) \longrightarrow S /\left(I\left(C_{n}^{k}\right), A_{n-k+i}\right) \longrightarrow 0 .
\end{aligned}
$$

By Lemma 4.1 we have $\left.S /\left(I\left(C_{n}^{k}\right), A_{n-1}\right)\right) \cong S_{n-k} / I\left(P_{n-k}^{k}\right)$. Now by Lemma 4.3 , we get

$$
S /\left(\left(I\left(C_{n}^{k}\right), A_{n-k+(i-1)}\right): x_{n-k+i}\right) \cong S_{n-2 k-1} / I\left(P_{n-2 k-1}^{k}\right)\left[x_{n-k+i}\right] .
$$

By Theorem 3.14 and Lemma 3.7, we obtain

$$
\begin{aligned}
\operatorname{sdepth}\left(S /\left(\left(I\left(C_{n}^{k}\right), A_{n-k+(i-1)}\right):\right.\right. & \left.\left.x_{n-k+i}\right)\right)= \\
& \left\lceil\frac{n-2 k-1}{2 k+1}\right\rceil+1=\left\lceil\frac{n}{2 k+1}\right\rceil .
\end{aligned}
$$


Again by Theorem 3.14, we have $\operatorname{sdepth}\left(S /\left(I\left(C_{n}^{k}\right), A_{n-1}\right)\right)=\left\lceil\frac{n-k}{2 k+1}\right\rceil$. By applying Lemma 2.4 on the above family of short exact sequences we get $\operatorname{sdepth}\left(S / I\left(C_{n}^{k}\right)\right) \geq\left\lceil\frac{n-k}{2 k+1}\right\rceil$.

Corollary 4.8. Let $n \geq 3$, if $n \geq 2 k+2$, then

$$
\begin{gathered}
\operatorname{sdepth}\left(S / I\left(C_{n}^{k}\right)\right)=\left\lceil\frac{n}{2 k+1}\right\rceil, \quad \text { if } n \equiv 0, k+1, \ldots, 2 k(\bmod (2 k+1)) ; \\
\left\lceil\frac{n}{2 k+1}\right\rceil-1 \leq \operatorname{sdepth}\left(S / I\left(C_{n}^{k}\right)\right) \leq\left\lceil\frac{n}{2 k+1}\right\rceil, \quad \text { if } n \equiv 1, \ldots, k(\bmod (2 k+1)) .
\end{gathered}
$$

Proof. When $k=1$, then by [6, Theorem 1.9], $\operatorname{sdepth}\left(S / I\left(C_{n}^{k}\right)\right) \leq\left\lceil\frac{n}{3}\right\rceil$. By Theorem 4.7 it is enough to prove that $\operatorname{sdepth}\left(S / I\left(C_{n}^{k}\right)\right) \leq\left\lceil\frac{n}{2 k+1}\right\rceil$ for $k \geq 2$ and $n \geq 2 k+2$. Since $x_{n-k} \notin I\left(C_{n}^{k}\right)$, thus by [4, Proposition 2.7] we have

$$
\operatorname{sdepth}\left(S / I\left(C_{n}^{k}\right)\right) \leq \operatorname{sdepth}\left(S /\left(I\left(C_{n}^{k}\right): x_{n-k}\right)\right) .
$$

Now we consider two cases:

(1) Let $2 k+2 \leq n \leq 3 k+1$, then $S /\left(I\left(C_{n}^{k}\right): x_{n-k}\right)=S /\left(I\left(P_{n}^{k}\right): x_{n-k}\right)$ so by the proof of Theorem 3.14 we have $\operatorname{sdepth}\left(S /\left(I\left(P_{n}^{k}\right): x_{n-k}\right)\right)=2=$ $\left\lceil\frac{n}{2 k+1}\right\rceil$. Therefore

$$
\operatorname{sdepth}\left(S / I\left(C_{n}^{k}\right)\right) \leq \operatorname{sdepth}\left(S /\left(I\left(C_{n}^{k}\right): x_{n-k}\right)\right)=2=\left\lceil\frac{n}{2 k+1}\right\rceil .
$$

(2) Let $n \geq 3 k+2$, then by Lemma 4.2

$$
S /\left(I\left(C_{n}^{k}\right): x_{n-k}\right) \cong S_{n-2 k-1} / I\left(P_{n-2 k-1}^{k}\right)\left[x_{n-k}\right] .
$$

By Lemma 3.7 and Theorem 3.14, $\operatorname{sdepth}\left(S_{n-2 k-1} / I\left(P_{n-2 k-1}^{k}\right)\left[x_{n-k}\right]\right)=$ $\left\lceil\frac{n}{2 k+1}\right\rceil$. Thus $\operatorname{sdepth}\left(S / I\left(C_{n}^{k}\right)\right) \leq \operatorname{sdepth}\left(S /\left(I\left(C_{n}^{k}\right): x_{n-k}\right)\right)=\left\lceil\frac{n}{2 k+1}\right\rceil$.

\section{Lower bounds for Stanley depth of edge ideals of $k^{\text {th }}$ powers of paths and cycles and a conjecture of Herzog}

In this section we compute some lower bounds for Stanley depth of $I\left(P_{n}^{k}\right)$ and $I\left(C_{n}^{k}\right)$. These bounds are good enough to prove that Conjecture 1.1 is true for $I\left(P_{n}^{k}\right)$ and $I\left(C_{n}^{k}\right)$. Let $0 \leq i \leq k-1$, define

$$
R_{n-k+i}:=K\left[\left\{x_{1}, x_{2}, \ldots, x_{n}\right\} \backslash\left\{x_{n-k}, x_{n-k+1}, \ldots, x_{n-k+i}\right\}\right]
$$


and

$$
B_{n-k+i}^{\prime}:=\left(x_{j}: x_{j} \in N_{P_{n}^{k}}\left(x_{n-k+i}\right) \backslash\left\{x_{n-k}, x_{n-k+1}, \ldots, x_{n-k+(i-1)}\right\}\right) .
$$

Thus $R_{n-k+i}$ is a subring of $S$ and $B_{n-k+i}^{\prime}$ is a monomial prime ideal of $S$. Let $I \subset Z=K\left[x_{i_{1}}, x_{i_{1}}, \ldots, x_{i_{r}}\right]$ be a monomial ideal and $Z^{\prime}:=Z\left[x_{i_{r}+1}\right]$. Then we write $I Z^{\prime}=I\left[x_{i_{r}+1}\right]$. Now we recall a useful remark of Cimpoeas.

Remark 5.1. [4, Remark 1.7] Let $I$ be a monomial ideal of $S$, and $I^{\prime}=$ $\left(I, x_{n+1}, x_{n+2}, \ldots, x_{n+m}\right)$ be a monomial ideal of $S^{\prime}=S\left[x_{n+1}, x_{n+2}, \ldots, x_{n+m}\right]$. Then

$$
\operatorname{sdepth}_{S^{\prime}}\left(I^{\prime}\right) \geq \min \left\{\operatorname{sdepth}_{S}(I)+m, \operatorname{sdepth}_{S}(S / I)+\left\lceil\frac{m}{2}\right\rceil\right\} .
$$

Theorem 5.2. Let $n \geq 2$, then $\operatorname{sdepth}\left(I\left(P_{n}^{k}\right)\right) \geq\left\lceil\frac{n}{2 k+1}\right\rceil+1$.

Proof. (a) If $n \leq 2 k+1$, then as the minimal generators of $I\left(P_{n}^{k}\right)$ have degree 2 , by $\left[15\right.$, Lemma 2.1] we have $\operatorname{sdepth}\left(I\left(P_{n}^{k}\right)\right) \geq 2=\left\lceil\frac{n}{2 k+1}\right\rceil+1$.

(b) For $n \geq 2 k+2$, if $k=1$, then by [19, Theorem 2.3], $\operatorname{sdepth}\left(I\left(P_{n}^{1}\right)\right) \geq$ $n-\left\lfloor\frac{n-1}{2}\right\rfloor=\left\lceil\frac{n-1}{2}\right\rceil+1 \geq\left\lceil\frac{n}{3}\right\rceil+1$. Now for $k \geq 2$, we prove this result by induction on $n$. We consider the following decomposition of $I\left(P_{n}^{k}\right)$ as a vector space:

$$
I\left(P_{n}^{k}\right)=I\left(P_{n}^{k}\right) \cap R_{n-k} \oplus x_{n-k}\left(I\left(P_{n}^{k}\right): x_{n-k}\right) S .
$$

Similarly, we can decompose $I\left(P_{n}^{k}\right) \cap R_{n-k}$ as follows:

$I\left(P_{n}^{k}\right) \cap R_{n-k}=I\left(P_{n}^{k}\right) \cap R_{n-k+1} \oplus x_{n-k+1}\left(I\left(P_{n}^{k}\right) \cap R_{n-k}: x_{n-k+1}\right) R_{n-k}$.

Continuing in the same way for $1 \leq i \leq k-1$ we have

$$
\begin{aligned}
I\left(P_{n}^{k}\right) \cap R_{n-k+i}= & I\left(P_{n}^{k}\right) \cap R_{n-k+(i+1)} \oplus \\
& x_{n-k+(i+1)}\left(I\left(P_{n}^{k}\right) \cap R_{n-k+i}: x_{n-k+(i+1)}\right) R_{n-k+i} .
\end{aligned}
$$

Finally we get the following decomposition of $I\left(P_{n}^{k}\right)$ :

$$
\begin{aligned}
& I\left(P_{n}^{k}\right)=I\left(P_{n}^{k}\right) \cap R_{n-1} \oplus \\
& \oplus_{i=1}^{k-1} x_{n-k+i}\left(I\left(P_{n}^{k}\right) \cap R_{n-k+(i-1)}: x_{n-k+i}\right) R_{n-k+i} \oplus x_{n-k}\left(I\left(P_{n}^{k}\right): x_{n-k}\right) S .
\end{aligned}
$$

Therefore

$$
\begin{gathered}
\operatorname{sdepth}\left(I\left(P_{n}^{k}\right)\right) \geq \min \left\{\operatorname{sdepth}\left(I\left(P_{n}^{k}\right) \cap R_{n-1}\right), \operatorname{sdepth}\left(\left(I\left(P_{n}^{k}\right): x_{n-k}\right) S\right),\right. \\
\left.\min _{i=1}^{k-1}\left\{\operatorname{sdepth}\left(\left(I\left(P_{n}^{k}\right) \cap R_{n-k+(i-1)}: x_{n-k+i}\right) R_{n-k+i}\right)\right\}\right\} .
\end{gathered}
$$


As $I\left(P_{n}^{k}\right) \cap R_{n-1}=\mathcal{G}\left(I\left(P_{n-k-1}^{k}\right)\right)\left[x_{n}\right]$, thus by induction on $n$ and Lemma 3.7 we have $\operatorname{sdepth}\left(I\left(P_{n}^{k}\right) \cap R_{n-1}\right) \geq\left\lceil\frac{n-k-1}{2 k+1}\right\rceil+1+1 \geq\left\lceil\frac{n}{2 k+1}\right\rceil+1$. Now we need to show that $\operatorname{sdepth}\left(\left(I\left(P_{n}^{k}\right): x_{n-k}\right) S\right) \geq\left\lceil\frac{n}{2 k+1}\right\rceil+1$ and

$$
\operatorname{sdepth}\left(\left(I\left(P_{n}^{k}\right) \cap R_{n-k+(i-1)}: x_{n-k+i}\right) R_{n-k+i}\right) \geq\left\lceil\frac{n}{2 k+1}\right\rceil+1 .
$$

For this we consider the following cases:

(1) Let $2 k+2 \leq n \leq 3 k+1$. If $n=2 k+2$, then $\left(I\left(P_{n}^{k}\right): x_{n-k}\right) S=$ $\left(x_{2}, \ldots, x_{n-k-1}, x_{n-k+1}, \ldots, x_{n}\right) S$, thus by [2, Theorem 2.2] and Lemma 3.7 we have

$$
\operatorname{sdepth}\left(\left(I\left(P_{n}^{k}\right): x_{n-k}\right) S\right)=\left\lceil\frac{n-2}{2}\right\rceil+2 \geq\left\lceil\frac{n}{2 k+1}\right\rceil+1 .
$$

If $2 k+3 \leq n \leq 3 k+1$, then by Remark 3.6, we get

$$
\left(I\left(P_{n}^{k}\right): x_{n-k}\right) S=\left(\mathcal{G}\left(I\left(P_{n-2 k-1}^{f(n-k)}\right)\right), B_{n-k}\right)\left[x_{n-k}\right] .
$$

Since $\operatorname{sdepth}\left(I\left(P_{n-2 k-1}^{f(n-k)}\right)\right)+\left|\mathcal{G}\left(B_{n-k}\right)\right| \geq 2$, by Remark 3.5 we have

$$
\operatorname{sdepth}\left(S_{n-2 k-1} / I\left(P_{n-2 k-1}^{f(n-k)}\right)\right)+\left\lceil\frac{\left|\mathcal{G}\left(B_{n-k}\right)\right|}{2}\right\rceil \geq 2,
$$

then by Remark 5.1, $\operatorname{sdepth}\left(\mathcal{G}\left(I\left(P_{n-2 k-1}^{f(n-k)}\right)\right), B_{n-k}\right) \geq 2$, and by Lemma 3.7 we have $\operatorname{sdepth}\left(\left(I\left(P_{n}^{k}\right): x_{n-k}\right) S\right) \geq 3=\left\lceil\frac{n}{2 k+1}\right\rceil+1$. Now since

$$
\begin{aligned}
\left(I\left(P_{n}^{k}\right) \cap R_{n-k+(i-1)}:\right. & \left.\left.x_{n-k+i}\right) R_{n-k+i}\right)= \\
( & \left.\left(I\left(P_{n-2 k-1+i}^{f(n-k+i)}\right)\right), B_{n-k+i}^{\prime}\right)\left[x_{n-k+i}\right] .
\end{aligned}
$$

So by the same arguments we have

$$
\operatorname{sdepth}\left(\left(I\left(P_{n}^{k}\right) \cap R_{n-k+(i-1)}: x_{n-k+i}\right) R_{n-k+i}\right) \geq 3=\left\lceil\frac{n}{2 k+1}\right\rceil+1 .
$$

(2) If $n \geq 3 k+2$, then by the proof of Lemma $3.3\left(I\left(P_{n}^{k}\right): x_{n-k}\right) S=$ $\left(\mathcal{G}\left(I\left(P_{n-2 k-1}^{k}\right)\right), B_{n-k}\right)\left[x_{n-k}\right]$ and

$$
\begin{aligned}
& \left(I\left(P_{n}^{k}\right) \cap R_{n-k+(i-1)}: x_{n-k+i}\right) R_{n-k+i}= \\
& \quad\left(\mathcal{G}\left(I\left(P_{n-2 k-1+i}^{k}\right)\right), B_{n-k+i}^{\prime}\right)\left[x_{n-k+i}\right] .
\end{aligned}
$$


By Remark 5.1 we have

$$
\begin{gathered}
\operatorname{sdepth}\left(\mathcal{G}\left(I\left(P_{n-2 k-1}^{k}\right)\right), B_{n-k}\right) \geq \min \left\{\operatorname{sdepth}\left(\mathcal{G}\left(I\left(P_{n-2 k-1}^{k}\right)\right)\right)+\right. \\
\left.\left|\mathcal{G}\left(B_{n-k}\right)\right|, \operatorname{sdepth}\left(S_{n-2 k-1} / I\left(P_{n-2 k-1}^{k}\right)\right)+\left\lceil\frac{\left|\mathcal{G}\left(B_{n-k}\right)\right|}{2}\right\rceil\right\}
\end{gathered}
$$

By induction on $n$ we have $\operatorname{sdepth}\left(\mathcal{G}\left(I\left(P_{n-2 k-1}^{k}\right)\right)\right) \geq\left\lceil\frac{n-2 k-1}{2 k+1}\right\rceil+$ $1=\left\lceil\frac{n}{2 k+1}\right\rceil$, and by Theorem 3.14, $\operatorname{sdepth}\left(S_{n-2 k-1} / I\left(P_{n-2 k-1}^{k}\right)\right)=$ $\left\lceil\frac{n}{2 k+1}\right\rceil-1$. Therefore $\operatorname{sdepth}\left(\mathcal{G}\left(I\left(P_{n-2 k-1}^{k}\right)\right), B_{n-k}\right) \geq\left\lceil\frac{n}{2 k+1}\right\rceil+1$. Thus by Lemma 3.7 we have $\operatorname{sdepth}\left(\left(I\left(P_{n}^{k}\right): x_{n-k}\right) S\right)>\left\lceil\frac{n}{2 k+1}\right\rceil+1$. Now using Remark 5.1 again, we get

$$
\begin{aligned}
& \operatorname{sdepth}(\mathcal{G}\left.\left(I\left(P_{n-2 k-1+i}^{k}\right)\right), B_{n-k+i}^{\prime}\right) \geq \\
& \min \left\{\operatorname{sdepth}\left(\mathcal{G}\left(I\left(P_{n-2 k-1+i}^{k}\right)\right)\right)+\left|\mathcal{G}\left(B_{n-k+i}^{\prime}\right)\right|\right. \\
&\left.\operatorname{sdepth}\left(S_{n-2 k-1+i} / I\left(P_{n-2 k-1+i}^{k}\right)\right)+\left\lceil\frac{\left|\mathcal{G}\left(B_{n-k+i}^{\prime}\right)\right|}{2}\right\rceil\right\} .
\end{aligned}
$$

By induction on $n$ we have $\operatorname{sdepth}\left(\mathcal{G}\left(I\left(P_{n-2 k-1+i}^{k}\right)\right)\right) \geq\left\lceil\frac{n-2 k-1+i}{2 k+1}\right\rceil+$ 1 , and by Theorem 3.14 we have $\operatorname{sdepth}\left(S_{n-2 k-1+i} / I\left(P_{n-2 k-1+i}^{k}\right)\right)=$ $\left\lceil\frac{n-2 k-1+i}{2 k+1}\right\rceil$. Therefore

$$
\operatorname{sdepth}\left(\mathcal{G}\left(I\left(P_{n-2 k-1+i}^{k}\right)\right), B_{n-k+i}^{\prime}\right) \geq\left\lceil\frac{n-2 k-1+i}{2 k+1}\right\rceil+1 .
$$

Thus by Lemma 3.7

$$
\operatorname{sdepth}\left(\left(I\left(P_{n}^{k}\right) \cap R_{n-k+(i-1)}: x_{n-k+i}\right) R_{n-k+i}\right) \geq\left\lceil\frac{n}{2 k+1}\right\rceil+1 .
$$

This completes the proof.

Proposition 5.3. Let $n \geq 2 k+1$, then $\operatorname{sdepth}\left(I\left(C_{n}^{k}\right) / I\left(P_{n}^{k}\right)\right) \geq\left\lceil\frac{n+k+1}{2 k+1}\right\rceil$.

Proof. When $k=1$, then by [6, Proposition 1.10] we have the required result. Now assume that $k \geq 2$ and consider the following cases:

(1). If $2 k+1 \leq n \leq 3 k+1$, then as $I\left(C_{n}^{k}\right)$ is a monomial ideal generated by degree 2 so by $\left[11\right.$, Theorem 2.1] $\operatorname{sdepth}\left(I\left(C_{n}^{k}\right) / I\left(P_{n}^{k}\right)\right) \geq 2=\left\lceil\frac{n+k+1}{2 k+1}\right\rceil$. 
(2). If $3 k+2 \leq n \leq 4 k+1$, then we use [11] to show that there exist Stanley decompositions of desired Stanley depth. Let $s \in\{1,2, \ldots k\}, j_{s} \in$ $\{1,2, \ldots, k+1-s\}$ and

$$
L:=\oplus_{s=1}^{k}\left(\oplus_{j_{s}=1}^{k+1-s} x_{j_{s}} x_{n+1-s} K\left[x_{j_{s}}, x_{j_{s}+k+1}, x_{n+1-s}\right]\right) .
$$

It is easy to see that $L \subset I\left(C_{n}^{k}\right) \backslash I\left(P_{n}^{k}\right)$. Now let $u_{i} \in I\left(C_{n}^{k}\right) \backslash I\left(P_{n}^{k}\right)$ be a squarefree monomial such that $u_{i} \notin L$ then clearly $\operatorname{deg}\left(u_{i}\right) \geq 3$. Since

$$
I\left(C_{n}^{k}\right) / I\left(P_{n}^{k}\right) \cong L \oplus_{u_{i}} u_{i} K\left[\operatorname{supp}\left(u_{i}\right)\right]
$$

Thus $\operatorname{sdepth}\left(I\left(C_{n}^{k}\right) / I\left(P_{n}^{k}\right)\right) \geq 3=\left\lceil\frac{n+k+1}{2 k+1}\right\rceil$ as required.

(3). If $n \geq 4 k+2$, then we have the following $K$-vector space isomorphism:

$$
\begin{gathered}
I\left(C_{n}^{k}\right) / I\left(P_{n}^{k}\right) \cong \\
\oplus_{j_{1}=1}^{k} x_{j_{1}} x_{n} \frac{K\left[x_{j_{1}+k+1}, x_{j_{1}+k+2}, \ldots, x_{n-k-1}\right]}{\left(x_{j_{1}+k+1} x_{j_{1}+k+2}, x_{j_{1}+k+1} x_{j_{1}+k+3}, \ldots, x_{n-k-2} x_{n-k-1}\right)}\left[x_{j_{1}}, x_{n}\right] \oplus \\
\oplus_{j_{2}=1}^{k-1} x_{j_{2}} x_{n-1} \frac{K\left[x_{j_{2}+k+1}, x_{j_{2}+k+2}, \ldots, x_{n-k-2}\right]}{\left(x_{j_{2}+k+1} x_{j_{2}+k+2}, x_{j_{2}+k+1} x_{j_{1}+k+3}, \ldots, x_{n-k-3} x_{n-k-2}\right)}\left[x_{j_{2}}, x_{n-1}\right] \oplus \\
\cdot \\
\cdot \\
\oplus_{j_{k-1}=1}^{2} x_{j_{k-1}} x_{n-k+2} \frac{K\left[x_{j_{k-1}+k+1}, x_{j_{k-1}+k+2}, \ldots, x_{n-2 k+1}\right]}{\left(x_{j_{k-1}+k+1} x_{j_{k-1}+k+2}, \ldots, x_{n-2 k} x_{n-2 k+1}\right)}\left[x_{j_{k-1}}, x_{n-k+2}\right] \oplus \\
x_{1} x_{n-(k-1)} \frac{K\left[x_{k+2}, x_{k+3}, \ldots, x_{n-2 k}\right]}{\left(x_{k+2} x_{k+3}, x_{k+2} x_{k+4}, \ldots, x_{n-2 k-1} x_{n-2 k}\right)}\left[x_{1}, x_{n-(k-1)}\right] .
\end{gathered}
$$

Thus

$$
\begin{gathered}
I\left(C_{n}^{k}\right) / I\left(P_{n}^{k}\right) \cong \\
\bigoplus_{s=1}^{k}\left(\oplus_{j_{s}=1}^{k+1-s} x_{j_{s}} x_{n+1-s}\left(S_{j_{s}+k+1, n-s-k} /\left(\mathcal{G}\left(I\left(P_{n}^{k}\right)\right) \cap S_{j_{s}+k+1, n-s-k}\right)\left[x_{j_{s}}, x_{n+1-s}\right]\right),\right.
\end{gathered}
$$

where $S_{j_{s}+k+1, n-s-k}=K\left[x_{j_{s}+k+1}, x_{j_{s}+k+2}, \ldots, x_{n-s-k}\right]$. Indeed, if $u \in I\left(C_{n}^{k}\right)$ such that $u \notin I\left(P_{n}^{k}\right)$ then $\left(x_{j_{s}} x_{n+1-s}\right) \mid u$ for only one pair of $s$ and $j_{s}$. If $\left(x_{j_{s}} x_{n+1-s}\right) \mid u$ then $u=x_{j_{s}}^{\gamma} x_{n+1-s}^{\delta} v$ and $v \in S_{j_{s}+k+1, n-s-k}$. Since $v \notin I\left(P_{n}^{k}\right)$, it follows that $v \notin \mathcal{G}\left(I\left(P_{n}^{k}\right)\right) \cap S_{j_{s}+k+1, n-s-k}$. Clearly

$$
S_{j_{s}+k+1, n-s-k} / \mathcal{G}\left(I\left(P_{n}^{k}\right)\right) \cap S_{j_{s}+k+1, n-s-k} \cong S_{n-\left(j_{s}+2 k+s\right)} / I\left(P_{n-\left(j_{s}+2 k+s\right)}^{k}\right) .
$$

Thus by Theorem 3.14 and Lemma 3.7, we have

$$
\operatorname{sdepth}\left(I\left(C_{n}^{k}\right) / I\left(P_{n}^{k}\right)\right) \geq \min _{s=1}^{k}\left\{\left\lceil\frac{n-\left(j_{s}+s+2 k\right)}{2 k+1}\right\rceil+2\right\} .
$$


It is easy to see that $\max \left\{j_{s}+s\right\}=k+1$. Therefore

$$
\operatorname{sdepth}\left(I\left(C_{n}^{k}\right) / I\left(P_{n}^{k}\right)\right) \geq\left\lceil\frac{n-(3 k+1)}{2 k+1}\right\rceil+2=\left\lceil\frac{n+k+1}{2 k+1}\right\rceil .
$$

Theorem 5.4. Let $n \geq 3$, then

$$
\begin{array}{ll}
\operatorname{sdepth}\left(I\left(C_{n}^{k}\right)\right) \geq 2, & \text { if } n \leq 2 k+1 \\
\operatorname{sdepth}\left(I\left(C_{n}^{k}\right)\right) \geq\left\lceil\frac{n-k}{2 k+1}\right\rceil+1, & \text { if } n \geq 2 k+2 .
\end{array}
$$

Proof. (a) If $n \leq 2 k+1$, then as the minimal generators of $I\left(C_{n}^{k}\right)$ have degree 2 , so by [15, Lemma 2.1] $\operatorname{sdepth}\left(I\left(C_{n}^{k}\right)\right) \geq 2$.

(b) If $n \geq 2 k+2$, then consider the short exact sequence

$$
0 \longrightarrow I\left(P_{n}^{k}\right) \longrightarrow I\left(C_{n}^{k}\right) \longrightarrow I\left(C_{n}^{k}\right) / I\left(P_{n}^{k}\right) \longrightarrow 0,
$$

by Lemma 2.4 we have

$$
\operatorname{sdepth}\left(I\left(C_{n}^{k}\right)\right) \geq \min \left\{\operatorname{sdepth}\left(I\left(P_{n}^{k}\right)\right), \operatorname{sdepth}\left(I\left(C_{n}^{k}\right) / I\left(P_{n}^{k}\right)\right)\right\}
$$

By Theorem 5.2, $\operatorname{sdepth}\left(I\left(P_{n}^{k}\right)\right) \geq\left\lceil\frac{n}{2 k+1}\right\rceil+1$, and by Proposition 5.3, we obtain $\operatorname{sdepth}\left(I\left(C_{n}^{k}\right) / I\left(P_{n}^{k}\right)\right) \geq\left\lceil\frac{n+k+1}{2 k+1}\right\rceil=\left\lceil\frac{n-k}{2 k+1}\right\rceil+1$.

Corollary 5.5. Let $n \geq 3$, if $n \leq 2 k+1$, then $\operatorname{sdepth}\left(I\left(C_{n}^{k}\right)\right) \geq 2=$ $\operatorname{sdepth}\left(S / I\left(C_{n}^{k}\right)\right)+1$. If $\bar{n} \geq 2 k+2$, then

$\operatorname{sdepth}\left(I\left(C_{n}^{k}\right)\right) \geq \operatorname{sdepth}\left(S / I\left(C_{n}^{k}\right)\right), \quad$ if $n \equiv 1, \ldots, k(\bmod (2 k+1))$; $\operatorname{sdepth}\left(I\left(C_{n}^{k}\right)\right) \geq \operatorname{sdepth}\left(S / I\left(C_{n}^{k}\right)\right)+1$, if $n \equiv 0, k+1, \ldots, 2 k(\bmod (2 k+1))$.

Proof. Proof follows by Corollary 4.8, Theorem 4.7 and Theorem 5.4.

\section{Acknowledgement}

The authors would like to thank the referee for a careful reading of the paper and for valuable comments. This research is partially supported by HEC Pakistan. 


\section{References}

[1] J. Apel, On a conjecture of R. P. Stanley; Part II Quotients modulo monomial ideals, J. Algebraic Combin., 17, (2003) 57-74.

[2] C. Biro, D. M. Howard, M. T. Keller, W. T. Trotter, S. J. Young, Interval partitions and Stanley depth, J. Combin. Theory, Ser. A, 117, (2010) 475482.

[3] J. A. Bondy, U. S. R. Murty, Graph Theory, Springer, 2008.

[4] M. Cimpoeas, Several inequalities regarding Stanley depth, Romanian Journal of Math. and Computer Science, 2, (2012) 28-40.

[5] M. Cimpoeas, Stanley depth of squarefree Veronese ideals, An. St. Univ. Ovidius Constanta, 21(3), (2013) 67-71.

[6] M. Cimpoeas, On the Stanley depth of edge ideals of line and cyclic graphs, Romanian Journal of Math. and Computer Science, 5(1), (2015) 70-75.

[7] M. Cimpoeas, Some remarks on the Stanley depth for multigraded modules, Le Matematiche LXIII, (2008) 165-171.

[8] A. M. Duval, B. Goeckneker, C. J. Klivans, J. L. Martine, A nonpartitionable Cohen-Macaulay simplicial complex, Adv. Math., 299, (2016) 381-395.

[9] J. Herzog, A survey on Stanley depth, In Monomial ideals, computations and applications, Lecture Notes in Math. Springer, Heidelberg, 2083, (2013) 3-45.

[10] J. Herzog, T. Hibi, Monomial Ideals, Springer-Verlag London Limited, (2011).

[11] J. Herzog, M. Vladoiu, X. Zheng, How to compute the Stanley depth of a monomial ideal, J. Algebra, 322(9), (2009) 3151-3169.

[12] J. Herzog, D. Popescu, M. Vladoiu, Stanley depth and size of a monomial ideal, Proc. Amer. Math. Soc., 140(2), (2012) 493-504.

[13] Z. Iqbal, M. Ishaq, M. Aamir, Depth and Stanley depth of edge ideals of square paths and square cycles, Comm. Algebra, 46(3), (2018), 1188-1198.

[14] M. Ishaq, M. I. Qureshi, Upper and lower bounds for the Stanley depth of certain classes of monomial ideals and their residue class rings, Comm. Algebra, 41(3), (2013) 1107-1116. 
[15] M. T. Keller, Y. Shen, N. Streib, S. J. Young, On the Stanley Depth of Squarefree Veronese Ideals, J. Algebraic Combin., 33(2), (2011) 313-324.

[16] M. T. Keller, S. J. Young, Combinatorial reductions for the Stanley depth of I and S/I, Electron. J. Combin. 24(3), (2017) \#P3.48.

[17] M. C. Lin, D. Rautenbach, F. J. Soulignac, J. L. Szwarcfiter, Powers of cycles, powers of paths, and distance graphs, Discrete Appl. Math., 159, (2011) 621-627.

[18] S. Morey, Depths of powers of the edge ideal of a tree, Comm. Algebra, 38(11), (2010) 4042-4055.

[19] R. Okazaki, A lower bound of Stanley depth of monomial ideals, J. Commut. Algebra, 3(1), (2011) 83-88.

[20] D. Popescu, M. I. Qureshi, Computing the Stanley depth, J. Algebra, 323(10),(2010) 2943-2959.

[21] D. Popescu, Stanley conjecture on intersection of four monomial prime ideals, Comm. Algebra, 41(11), (2013) 4351-4362.

[22] M. R. Pournaki, S. A. Seyed Fakhari, S. Yassemi, Stanley depth of powers of the edge ideals of a forest, Proc. Amer. Math. Soc., 141(10), (2013) 3327-3336.

[23] A. Rauf, Depth and Stanley depth of multigraded modules, Comm. Algebra, 38(2), (2010) 773-784.

[24] R. P. Stanley, Linear Diophantine equations and local cohomology, Invent. Math., 68(2), (1982) 175-193.

[25] A. Stefan, Stanley depth of powers of path ideal, http://arxiv.org/pdf/1409.6072.pdf.

[26] Z. Tang, Stanley depths of certain StanleyReisner rings, J. Algebra, 409(1), (2014) 430-443.

Zahid Iqbal,

School of Natural Sciences,

National University of Sciences and Technology Islamabad,

Sector H-12, Islamabad Pakistan.

Email: 786zahidwarraich@gmail.com

Muhammad Ishaq,

School of Natural Sciences,

National University of Sciences and Technology Islamabad,

Sector H-12, Islamabad Pakistan.

Email: ishaq_maths@yahoo.com 